\title{
Caractérisation physico-chimique et bactériologique des eaux souterraines de Bingerville
}

\author{
Fulvie KANOHIN Epse OTCHOUMOU ${ }^{1 *}$, Ossey Bernard YAPO $^{2}$, Brou DIBI $^{3}$ et \\ Aya Carole BONNY ${ }^{4}$ \\ ${ }^{1}$ Laboratoire Géosciences et Environnement (LGE), Université Nangui, Abrogoua, \\ 02 BP 801 Abidjan, Côte d'Ivoire. \\ ${ }^{2}$ Laboratoire des Sciences de l'Environnement (LES), Université Nangui, Abrogoua, \\ 02 BP 801 Abidjan, Côte d'Ivoire. \\ ${ }^{3}$ Laboratoire Géosciences et Environnement (LGE), Université Jean Lorougnon Guédé, \\ BP 150 Daloa, Côte d'Ivoire. \\ ${ }^{4}$ Laboratoire de Biotechnologies, UFR Biosciences, \\ Université Félix HOUPHOUET-BOIGNY Abidjan, 22 BP 582 Abidjan, Côte d'Ivoire. \\ *Auteur correspondant ; E-mail: atchoful@yahoo.fr
}

\section{RESUME}

L'accès à l'eau potable dans les zones rurales et semi-rurales constitue une préoccupation majeure pour les populations à cause de la qualité de la ressource. L'objectif de cette étude était d'évaluer le degré de pollution des eaux souterraines au Sud de la Côte d'Ivoire. La méthodologie adoptée a consisté à évaluer le niveau de pollution par le suivi de la variation spatiale des teneurs des indicateurs de pollution des eaux consommées. Des tests statistiques ont permis d'avoir l'origine probable des sources de pollution. Les paramètres de pollution les plus menaçants restent le phosphate $\left(\mathrm{PO}_{4}^{3-}\right)$ et l'ammonium $\left(\mathrm{NH}_{4}^{+}\right)$. Les résultats des tests statistiques révèlent deux phénomènes majeurs que sont la pollution d'origine superficielle représentée par le facteur 1 et la minéralisation au niveau des facteurs 2 et 3 . Les minéraux $\mathrm{PO}_{4}{ }^{3-}, \mathrm{NH}_{4}{ }^{+}$et $\mathrm{NO}_{3}{ }^{-}$ observés au niveau du facteur 1 montrent que ces ions qui menacent la qualité de ces ressources pourraient provenir d'activités humaines. Ces résultats sont confirmés par les tests bactériologiques à travers l'identification et le dénombrement des coliformes fécaux et totaux, des streptocoques fécaux et des clostridium perfringens. La présence de ces bactéries traduit une pollution d'origine fécale rendant ces eaux impropres à la consommation humaine.

(C) 2017 International Formulae Group. All rights reserved.

Mots clés : Eaux souterraines, sels dissous, pollution fécale, Bingerville.

\section{Physicochemical and bacteriological characterization of Bingerville groundwater}

\begin{abstract}
Rural and semi-rural areas drinking water access is a major concern of populations because of the resource quality. This study aimed at assessing the pollution degree of groundwater resources in southern Côte d'Ivoire. The methodology adopted consisted in assessing the pollution level by monitoring the spatial variation
\end{abstract}


in the water pollution indicators levels. Statistical analyses have led to the probable origin of pollution sources. The most threatening pollution parameters remain phosphate $\left(\mathrm{PO}_{4}{ }^{3-}\right)$ and ammonium $\left(\mathrm{NH}_{4}^{+}\right)$. The statistical analysis show two major phenomena: the surface pollution represented by the factor 1 and the mineralization factors 2 and 3. Minerals $\left(\mathrm{PO}_{4}{ }^{3-}, \mathrm{NH}_{4}^{+}\right.$and $\left.\mathrm{NO}_{3}{ }^{-}\right)$observed in Factor 1 show that ions that threaten these resources quality would come from human activities. Identification and bacteriological analysis of total fecal coliforms, Streptococci and Clostridium perfringens confirmed these results. These bacteria fecal pollution made the water resources unfit for human consumption.

(C) 2017 International Formulae Group. All rights reserved.

Keywords: Groundwater, dissolved salts, faecal pollution, Bingerville.

\section{INTRODUCTION}

L'eau est une ressource essentielle aux besoins fondamentaux de l'homme et à son environnement. Les eaux souterraines constituent une ressource en eau douce des collectivités rurales. Cependant, l'eau captée peut contenir des éléments pouvant avoir des effets indésirables sur la santé, comme des microorganismes pathogènes, des substances indésirables ou même des substances toxiques (Yapo et al., 2010 ; Jang et al., 2012). Ces substances peuvent provenir soit du milieu physique dans lequel l'eau a évolué, soit des rejets de certaines activités humaines dont l'eau est devenue le réceptacle. Des études menées en Côte d'Ivoire en milieu urbain par Coulibaly et al. (2004), Fofana (2005), Jourda et al. (2006), et Yapo et al. (2010) ont permis de caractériser les eaux de puits à usage domestique des quartiers précaires d'Abidjan et de mettre en évidence les sources de pollution de ces puits. Les résultats de ces études font état de pollution, chimique et bactériologique d'origine anthropique. La ville de Bingerville, comme celle d'Abidjan, exploite pour son alimentation en eau potable la nappe profonde. En milieu rural, les localités exploitent la nappe phréatique à travers les puits et les sources, l'accès aux services d'assainissement étant inexistant. L'évacuation des eaux usées et des excréta de ces villages est réalisée en grande majorité par des latrines traditionnelles à fonds perdu dont la fosse réalisée par des puisatiers de fortune pourrait atteindre la nappe phréatique et constitue un risque de contamination microbiologique des eaux souterraines, rendant la nappe vulnérable à la pollution. Cette pollution, générée par les activités anthropiques, peut, en atteignant des niveaux critiques, présenter un danger grave pour la santé publique (Ambarref et al., 2007). Il est donc à craindre que la qualité des eaux souterraines ne se dégrade de plus en plus dans ces localités où la quasi-totalité des ménages exploitent cette ressource pour satisfaire leurs besoins en eau. L'objectif de cette étude est de montrer la vulnérabilité des nappes phréatiques à travers la caractérisation de la qualité des eaux de puits et de sources à usages domestiques des villages de Brégbo et de Koffikro.

\section{MATERIEL ET METHODES \\ Présentation de la zone d'étude}

Les localités de Bregbo et Koffikro prospectées appartiennent à la sous-préfecture de Bingerville située au Sud de la Côte d'Ivoire (Figure 1). Bingerville couvre une superficie de $2119 \mathrm{~km}^{2}$ et sa population est estimée à 9131 habitants (INS, 2014). Au cours des prospections et des campagnes d'échantillonnages des puits dans les villages de Bingerville (Anan, Agban; Adjamébingerville, Danhokro, Elokaté, Elokato, Santè, Brègbo et Koffikro), le constat a été fait de la présence de châteaux d'eau fonctionnels, dans presque tous les villages. $\mathrm{Ce}$ qui n'était pas le cas pour Brègbo et Koffikro qui utilisaient les eaux de puits pour tous leurs besoins en eau. Ces deux villages ont été choisis pour analyser les dangers auxquels les populations ont été exposées en s'alimentant avec des eaux vulnérables à la pollution. Brégbo et koffikro sont situées à 9 $\mathrm{km}$ de Bingerville (Figure 2). Ces deux localités couvrent une superficie d'environ $100 \mathrm{~km}^{2}$ et leur population est estimée à 2584 
habitants (INS, 2014). La zone d'étude bénéficie d'un climat caractérisé par deux saisons de pluies alternées par deux saisons sèches avec une moyenne pluviométrique annuelle de $1800 \mathrm{~mm}$. Les pluies annuelles (2005-2015) de la station de Bingerville ont été obtenues auprès de la Société de Développement Aéroportuaire et Météorologique (SODEXAM). Bingerville appartient au bassin sédimentaire du Continental Terminal (sud du pays), et est essentiellement constituée de sables argileux et de grès datant du tertiaire, précisément du pliocène (Tagini, 1971). Sur le plan morphologique, cette région appartient à la zone dite des "hauts plateaux" qui occupe la partie Nord du système lagunaire, dont le substratum, formé de sables argileux, a engendré un relief aux formes douces et vallonnées, ou les accidents importants sont rares et les altitudes, inférieures à $100 \mathrm{~m}$ (ACI, 1986). Bingerville est drainée par deux cours d'eau, la lagune Aghien et la lagune Ebrié.

\section{Echantillonnage}

Les échantillons des eaux de puits et de source ont été prélevés à Brégbo et Koffikro. Ces prélèvements ont été effectués en saison des pluies (du 15 au 17 octobre et du 02 au 05 novembre 2015 ; du 22 au 26 mai et 10 au 12 juin 2016), périodes qui correspondent à la recharge de la nappe phréatique par les précipitations. Les échantillons d'eau prélevés sont mis dans des bouteilles en polyéthylène de capacité 1 litre, préalablement lavées à l'acide nitrique puis à l'eau distillée. Sur le terrain avant remplissage des bouteilles, celles-ci ont été lavées trois fois avec l'eau à prélever. Le remplissage des bouteilles a été fait à ras bord puis le bouchon vissé afin d'éviter tout échange gazeux avec l'atmosphère. Les prélèvements pour les analyses microbiologiques ont été effectués à l'aide de bouteilles préalablement autoclavées. Les échantillons ont été conservés en glacière réfrigérée pendant le transport jusqu'au laboratoire où les analyses ont été aussitôt effectuées. Les paramètres physiques ont été mesurés in situ: La température, la conductivité électrique, les Sels Dissous Totaux (TDS) et le pH. Le niveau dynamique de la nappe a été mesuré à chaque prélèvement. Pour chaque prélèvement, 14 paramètres ont été mesurés dont 11 physicochimiques et 3 paramètres microbiologiques. $\mathrm{Au}$ total, Une cinquantaine (50) de puits ont été échantillonnées lors des différentes campagnes dans les villages de Bingerville dont 20 puits pour Brègbo et Koffikro.

Analyses chimiques et bactériologiques

$\mathrm{Au}$ laboratoire, les ions majeurs $\left(\mathrm{Cl}^{-}\right.$, $\mathrm{SO}_{4}{ }^{2-}$ ), la dureté totale (TH) et les sels nutritifs ont été dosés. Les chlorures et l'alcalinité ont été dosés par titration (Rodier et al., 1996 ; AFNOR, 1997). Les ions $\mathrm{SO}_{4}{ }^{2-}$ ont été dosés par néphélométrie. Les dérivés azotés, par contre, ont été mesurés par colorimétrie à l'aide d'un spectrophotomètre Hach DR 2010, nitrates $\left(\mathrm{NO}_{3}{ }^{-}\right)$par réduction au cadmium, les nitrites $\left(\mathrm{NO}_{2}^{-}\right)$par diazotation et l'ammonium $\left(\mathrm{NH}_{4}^{+}\right)$par la méthode à l'indophénol. Les analyses microbiologiques ont permis d'identifier et de dénombrer les Streptocoques fécaux, les Coliformes totaux, Coliformes fécaux et les Clostridium perfringens. Ces microorganismes ont été identifiés et dénombrés en filtrant des aliquotes homogènes de $100 \mathrm{ml}$ sur une membrane dont le diamètre des pores est de $0,45 \mu \mathrm{m}$. Les membranes ont ensuite été placées sur des milieux sélectifs pendant 24 heures à $37^{\circ} \mathrm{C}$ à l'étuve thermo-statée. Les milieux suivants ont été utilisés: la gélose KF (Milieu sélectif utilisé pour l'isolement et le dénombrement des entérocoques dans les produits alimentaires par la méthode classique de numération en boîtes de Pétri) pour les Streptocoques fécaux, le coli ID (milieu de culture pour l'identification d'Escherichia coli) pour les coliformes totaux, la gélose TSN (Tryptone Sulfite Néomycine) pour le Clostridium sulfito-réducteur dont le plus marquant est le Clostridium perfringens.

\section{Analyses statistiques}

L'analyse statistique utilisée est basée sur l'analyse en composante principale (ACP). L'ACP est une méthode statistique (initialement de statistique descriptive) qui a 
pour but de comprendre et de visualiser comment les effets de phénomènes a priori isolés se combinent (Caillez et Pages, 1976). C'est une méthodologie largement utilisée pour interpréter les données hydrochimiques (El Asslouj et al., 2007 ; Yapo et al., 2010 ). L'analyse statistique a été effectuée avec 19 échantillons et de 13 variables (température, $\mathrm{pH}$, conductivité, $\mathrm{NH}_{4}^{+}, \mathrm{PO}_{4}{ }^{3-}, \mathrm{SO}_{4}{ }^{2-}, \mathrm{Cl}^{-}$, $\mathrm{Ca}^{2+}, \mathrm{Mg}^{2+}, \mathrm{K}^{+}, \mathrm{Na}^{+}, \mathrm{NO}_{3}{ }^{-}$et $\left.\mathrm{NO}_{2}{ }^{-}\right)$. Les tests statistiques ont été effectués pour connaître les sources de pollutions des eaux souterraines.
D'abord une analyse simplifiée des paramètres étudiés en comparaison avec les normes OMS a été faite. Ensuite l'étude de la typologie de la pollution des eaux de puits a été faite à l'aide d'une Analyse en Composante Principale Normées (ACPN). Les valeurs propres, les cartes factorielles et les cercles de corrélations ont été obtenus avec le logiciel Statistica 7.1. Ce qui a permis de traiter des caractères numériques jouant tous le même rôle (Bourouche et Saporta, 1983).

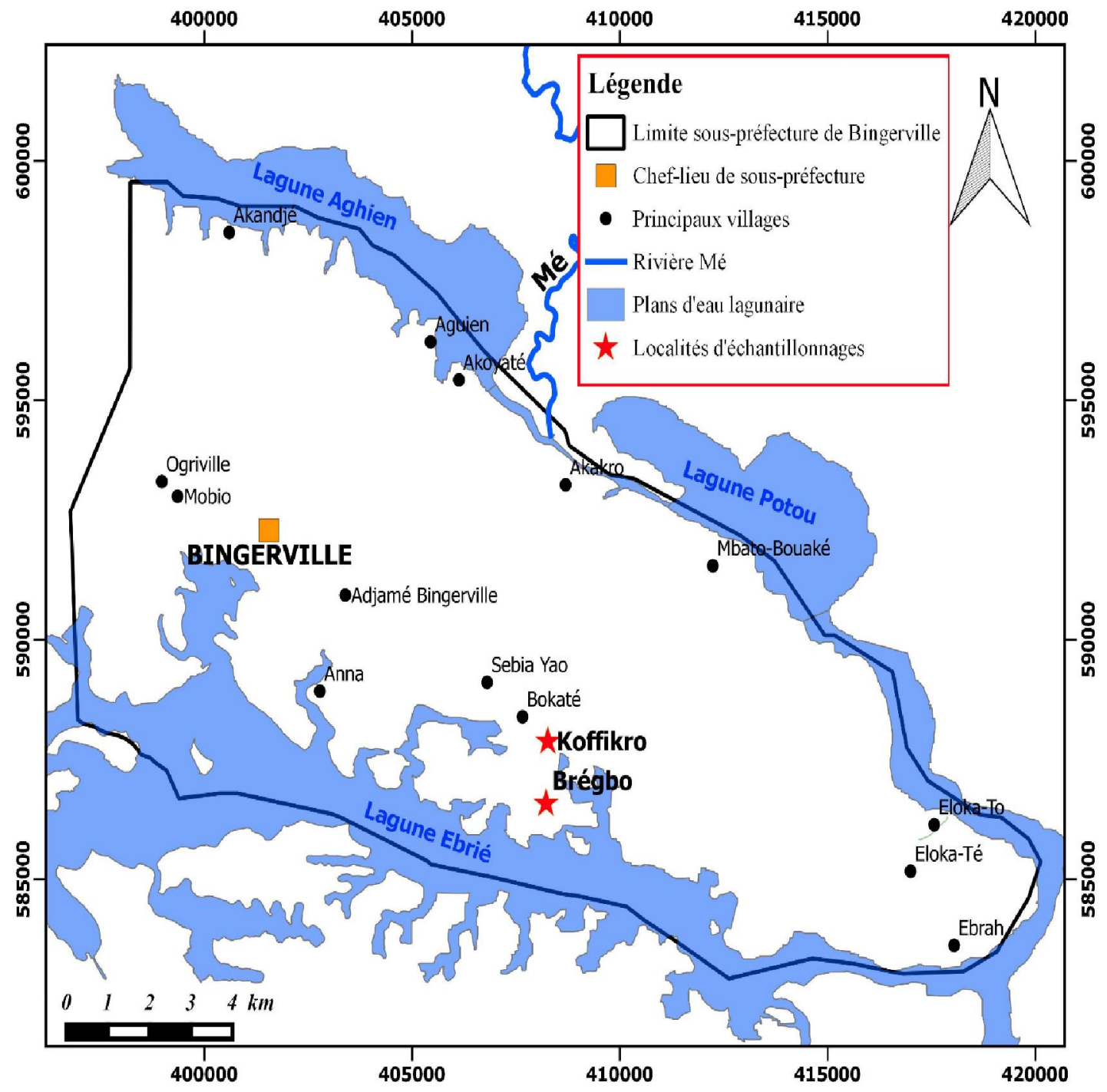

Figure 1: Situation géographique de Bingerville. 


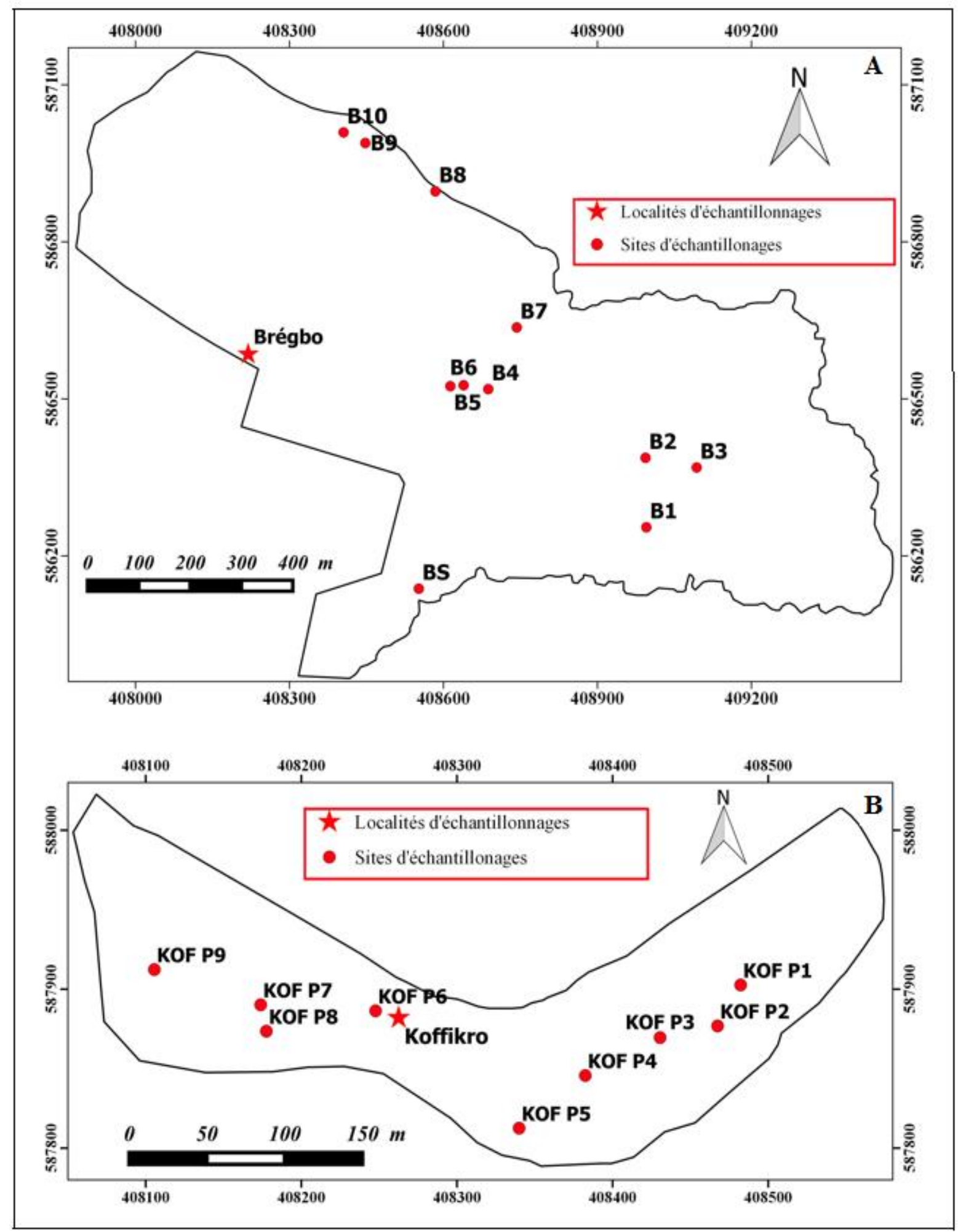

Figure 2 : Présentation des localités échantillonnées : Brègbo (A) et Koffikro (B). 
RESULTATS

\section{Caractéristiques des puits étudiés}

Les villages de Brègbo et de Koffikro sont assez particuliers car au cours de la campagne de prélèvement, le château d'eau de Brègbo n'était pas encore mis en service, et la zone de Koffikro échantillonnée est peuplée par des allogènes (burkinabés et maliens) qui pratiquent l'élevage de porcs et utilisent les mêmes points d'eau que les populations. Ces deux villages au moment de la campagne d'échantillonnage s'alimentaient exclusivement avec les eaux des puits étudiés

Les puits étudiés ont des profondeurs variant entre $0,5 \mathrm{~m}$ et $9,00 \mathrm{~m}$. Dans le village de Brègbo, les profondeurs des puits varient entre $1,75 \mathrm{~m}$ et $8,38 \mathrm{~m}$ avec les niveaux piézométriques qui oscillent entre $1,36 \mathrm{~m}$ et $8,02 \mathrm{~m}$. Les profondeurs des puits de Koffikro varient entre $3,88 \mathrm{~m}$ et $7,73 \mathrm{~m}$ avec des niveaux d'eaux variant entre 2,8 et $6,18 \mathrm{~m}$. Cette proximité de l'eau souterraine par rapport à la surface topographique renforce le caractère vulnérable de la plupart des puits des différentes localités de Bingerville. L'accès au service d'assainissement moderne étant inexistant en zone rurale, la plupart de ces points d'eau sont côtoyés par les ouvrages d'assainissement autonome abandonnés ou en usage. La distance moyenne entre les latrines et les différents points d'eaux est inférieur à $10 \mathrm{~m}$. Les ouvrages d'assainissement sont parfois en amont des puits d'eau.

\section{Caractérisation physico-chimique des eaux}

Les paramètres physiques et chimiques comprennent la température, le $\mathrm{pH}$, la conductivité, le TDS, l'oxygène dissous, l'ammonium, le phosphate, les nitrates, le sodium, le calcium et le magnésium.

Dans la zone d'étude, la température ne présente pas de grandes variations d'un puits à l'autre (Figure 3B) et reste voisine de la température moyenne annuelle de la région soit $27{ }^{\circ} \mathrm{C}$, avec un minimum de $26,14^{\circ} \mathrm{C}$ à Koffikro et un maximum de $28,48{ }^{\circ} \mathrm{C}$ à Brègbo.

Dans ces deux localités, le pH varie entre 3,72 et 6,14 (Figure 3A). Les eaux de cette région ont une tendance acide. L'acidité est d'autant plus importante dans les puits de Koffikro.

La conductivité électrique quant à elle est comprise entre $72 \mu \mathrm{S} / \mathrm{cm}$ et $860 \mu \mathrm{S} / \mathrm{cm}$ (Figure 3C). Les eaux des puits B2, B4, B6, $\mathrm{B} 7, \mathrm{~K} 1, \mathrm{~K} 2, \mathrm{~K} 3$ et $\mathrm{K} 4$ ont une conductivité électrique supérieure à la valeur guide de l'OMS qui est de $300 \mu \mathrm{S} / \mathrm{cm}$, soit $40 \%$ des points d'eaux analysées. Certains points d'eaux sont très faiblement minéralisés avec une conductivité inférieure à $90 \mu \mathrm{S} / \mathrm{cm}$; c'est le cas de la source d'eau du village de Brègbo $(86 \mu \mathrm{S} / \mathrm{cm})$ et du puits K9 de Koffikro (72 $\mu \mathrm{S} / \mathrm{cm})$. Les fortes valeurs de conductivité ont été constatés au niveau des puits B7, K1, K2 et $\mathrm{K} 3$ dont les valeurs respectives sont de 764 $\mu \mathrm{S} / \mathrm{cm}, 860 \mu \mathrm{S} / \mathrm{cm}, 495 \mu \mathrm{S} / \mathrm{cm}$ et $744 \mu \mathrm{S} / \mathrm{cm}$.

Les concentrations en ions $\mathrm{NH}_{4}{ }^{+}, \mathrm{PO}_{4}{ }^{3-}$ , $\mathrm{SO}_{4}{ }^{2-}$ et $\mathrm{NO}_{3}{ }^{-}$et les valeurs seuils dans les puits de Brègbo et Koffikro sont indiquées dans le Tableau 1. Ces paramètres sont des indicateurs de pollution pouvant mettre en cause la qualité des eaux à usage domestique. Les concentrations des eaux de puits à Brègbo en ammonium sont inférieures à $0,2 \mathrm{mg} / \mathrm{L}$. Ce qui n'est pas le cas à Koffikro où l'on observe des pics d'ammonium allant jusqu'à $3,9 \mathrm{mg} / \mathrm{L}$. Les concentrations en phosphates sont élevées dans tous les puits de koffikro et sept puits sur onze à Brègbo ont également leur concentration en phosphate élevée. Les valeurs de ce paramètre dans les eaux étudiées sont très variables et oscillent entre 0,004 $\mathrm{mg} / \mathrm{L}$ et $0,29 \mathrm{mg} / \mathrm{l}$. L'analyse des teneurs en nitrates des puits montre une légère variation qui oscille entre $0,7 \mathrm{mg} / 1$ et $11,2 \mathrm{mg} / \mathrm{l}$ et qui restent inférieures à la valeur seuil de l'OMS $(50 \mathrm{mg} / \mathrm{l})$. De ce fait, les eaux étudiées ne sont pas assujetties à un risque de pollution par les nitrates.

\section{Analyse en composante principale (ACP) de toutes les eaux}

Les résultats de l'analyse statistique (ACP) donnent de nombreux tableaux dont certains sont résumés dans cette étude. Le tableau des valeurs propres (Tableau 2) montre que les trois premiers facteurs 
représentent $74,83 \%$ de la variance exprimée. Ils peuvent donc permettre d'interpréter les résultats obtenus. La matrice de corrélation (Tableau 3) montre les différentes corrélations entre les paramètres physico-chimiques étudiés. Cette matrice met en évidence des corrélations significatives entre la conductivité, les nitrites, les nitrates, le sulfate, le potassium, le sodium et les ions chlorures. La matrice révèle également une corrélation entre le magnésium et le calcium ( $\mathrm{r}$ $=0,811)$; une corrélation entre le potassium et le sodium $(\mathrm{r}=0,814)$ et entre les ions chlorures et le sulfate $(\mathrm{r}=0,522)$.

L'analyse des plans factoriels (Figure 4) révèle également une corrélation entre les paramètres physico-chimique des eaux étudiées. Ainsi, le facteur F1 dans les plans factoriels F1-F2 et F1-F3 est déterminé par la conductivité, le potassium, le sodium, le sulfate, le nitrate, le magnésium, le calcium, et la température. Cet axe peut être assimilé au caractère minéral des eaux dont dépends les paramètres indiqués ci-dessous. L'axe F3 dans le plan factoriel F1-F3 est défini par l'ammonium, le phosphate et les ions chlorures. Ce facteur exprime la pollution des eaux par les activités anthropiques et l'intrusion des eaux saumâtres dans la nappe phréatique. Le facteur F2 dans l'axe F1-F2 est défini par le $\mathrm{pH}$, le phosphate, le calcium et le magnésium.

La carte factorielle des puits (Figure 5) permet de regrouper ces eaux en quatre classes. La classe 1 est caractérisée par des eaux de puits fortement minéralisées. Les concentrations en sels minéraux sont élevées $\left(\mathrm{SO}_{4}{ }^{2-}, \mathrm{Cl}^{-}, \mathrm{K}^{+}\right.$et $\left.\mathrm{Na}^{+}\right)$et fortement affectées par l'activité anthropique. La conductivité des puits de cette classe est comprise entre 497 $\mu \mathrm{S} / \mathrm{cm}$ et $860 \mu \mathrm{S} / \mathrm{cm}$ avec des concentrations d'ammonium et de phosphate élevées. Ces eaux ont les $\mathrm{pH}$ les plus faibles. Le $\mathrm{pH}$ est compris entre 3,72 et 3,75 . On retrouve ces puits essentiellement à Koffikro (K1, K2 et K3).

La classe C2 est caractérisée par des eaux faiblement minéralisées. Les concentrations des sels minéraux sont faibles avec une conductivité comprise entre 72 $\mu \mathrm{S} / \mathrm{cm}$ et $341 \mu \mathrm{S} / \mathrm{cm}$. Les concentrations d'ammonium dans ces puits sont inférieures à $0,5 \mathrm{mg} / \mathrm{l}$ avec des taux de phosphate supérieur à $0,005 \mathrm{mg} / \mathrm{l}$ (la valeur seuil de l'OMS)

La classe C3 est caractérisée par des eaux moyennement minéralisées avec une conductivité comprise entre $215 \mu \mathrm{S} / \mathrm{cm}$ et 764 $\mu \mathrm{S} / \mathrm{cm}$. Les concentrations d'ammonium dans ces puits sont inférieures à $0,5 \mathrm{mg} / 1$ et $40 \%$ des puits de cette classe présentent des taux de phosphate supérieur à $0,005 \mathrm{mg} / \mathrm{l}$ (la valeur seuil de l'OMS). Ces puits sont beaucoup affectés par les activités anthropiques (proximités des fosses septiques, présence des décharges d'ordures ménagères) et infiltration des eaux saumâtres en provenance de la lagune à proximité du village de Brègbo

La classe $\mathrm{C} 4$ est caractérisée par des eaux faiblement minéralisées avec des concentrations en ammonium et en phosphate faibles et moins affectés par l'activité anthropique.

\section{Caractérisation microbiologique des eaux}

Les analyses microbiologiques montrent la présence des bactéries de types coliformes totaux, coliformes fécaux, streptocoques fécaux et Clostridium perfringens dans les eaux souterraines (Figure 6). Les charges moyennes des Clostridiums, des coliformes et streptocoques sont exprimées dans le Tableau 4. Les coliformes totaux sont présents dans tous les puits des localités visitées. Les concentrations de coliformes totaux varient de $200 \mathrm{UFC} / 100 \mathrm{ml}$ à $39600 \mathrm{UFC} / 100 \mathrm{ml}$ avec une moyenne de $11600 \mathrm{UFC} / 100 \mathrm{ml}$ à Koffikro et 200 $\mathrm{UFC} / 100 \mathrm{ml}$ à $2500 \mathrm{UFC} / 100$ avec une moyenne de $1318 \mathrm{UFC} / 100 \mathrm{ml}$ à Brègbo. Les concentrations de coliformes fécaux varient de 0 à $16000 \mathrm{UFC} / 100 \mathrm{ml}$ avec une moyenne de 3311 UFC/100 ml à Koffikro et de 0 à 1700 $\mathrm{UFC} / 100 \mathrm{ml}$ à Brègbo avec une moyenne de $620 \mathrm{UFC} / 100 \mathrm{ml}$. Ces bactéries sont de bons indicateurs de contaminations fécales récentes. Tous les puits visités sont contaminés par les coliformes fécaux sauf le puits B1. Les streptocoques fécaux sont moins 
abondants dans les eaux analysées. Leurs concentrations varient de $20 \mathrm{UFC} / 100 \mathrm{ml}$ à $360 \mathrm{UFC} / 100 \mathrm{ml}$ avec une moyenne de 84 $\mathrm{UFC} / 100 \mathrm{ml}$ à Brègbo et de 0 à 6800 $\mathrm{UFC} / 100 \mathrm{ml}$ avec une moyenne de 2113 UFC/100 $\mathrm{ml}$ à Koffikro. Ces bactéries sont également de bons indicateurs de contamination fécale. Les Clostridium perfringens sont présent dans 7 puits sur 20 puits soit $35 \%$ des puits (Figure 7). Les concentrations varient de 0 à $8 \mathrm{UFC} / 20 \mathrm{ml}$ soit $40 \mathrm{UFC} / 100 \mathrm{ml}$ avec une moyenne de 10 $\mathrm{UFC} / 100 \mathrm{ml}$ à Bregbo, et de 0 à $150 \mathrm{UFC} / 100$ $\mathrm{ml}$ pour une moyenne de $30 \mathrm{UFC} / 100 \mathrm{ml}$ à Koffikro.
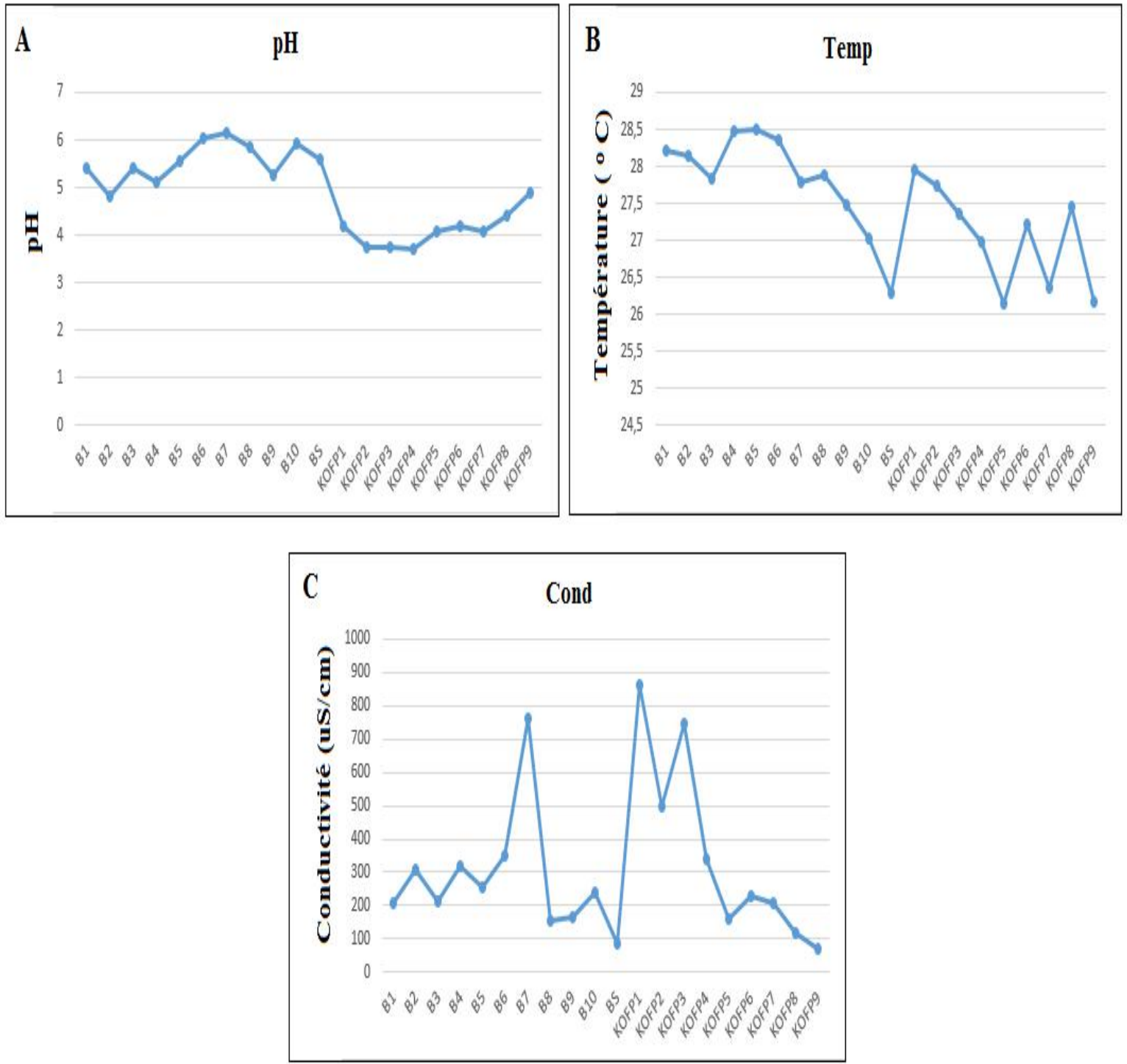

Figure 3: Evolution spatiale des paramètres physiques pH; temperature; conductivité à Brègbo et Koffikro. 
Tableau 1 : Sommaire statistique des concentrations des ions indicateurs de pollution des puits de Brègbo et Koffikro.

\begin{tabular}{lcccc}
\hline Ions & Min $(\mathrm{mg} / \mathrm{l})$ & Moy $(\mathrm{mg} / \mathrm{l})$ & Max $(\mathrm{mg} / \mathrm{l})$ & Valeur limite $(\mathrm{mg} / \mathrm{l})$ \\
\hline $\mathrm{NH}_{4}{ }^{+}$ & $<0,01$ & 0,365 & 3,9 & 0,5 \\
$\mathrm{PO}_{4}{ }^{3-}$ & $<0,004$ & 0,08 & 0,29 & 0,005 \\
$\mathrm{NO}_{3}{ }^{-}$ & 0,7 & 4,5 & 11,2 & 50 \\
$\mathrm{SO}_{4}{ }^{2-}$ & 0,0 & 23,56 & 64,81 & 250 \\
\hline
\end{tabular}

Tableau 2 : Valeurs propres et pourcentages des variances exprimées par les axes principaux.

\begin{tabular}{lcccc}
\hline & Val Propre & $\begin{array}{c}\text { \% Total } \\
\text { variance }\end{array}$ & $\begin{array}{c}\text { Cumul } \\
\text { Val Propre }\end{array}$ & $\begin{array}{c}\text { Cumul } \\
\text { \% }\end{array}$ \\
\hline F1 & 5,236622 & 40,28171 & 5,23662 & 40,2817 \\
F2 & 2,708868 & 20,83744 & 7,94549 & 61,1192 \\
F3 & 1,783272 & 13,71748 & 9,72876 & 74,8366 \\
\hline
\end{tabular}

Tableau 3 : Corrélations entre les paramètres physico-chimique des eaux.

\begin{tabular}{|c|c|c|c|c|c|c|c|c|c|c|c|c|c|}
\hline & NO2 & $\mathrm{NH} 4$ & PO4 & NO3 & $\mathrm{pH}$ & $\mathrm{T}^{\circ} \mathrm{C}$ & SO4 & $\mathrm{Cl}$ - & $\mathrm{Ca} 2+$ & $\mathrm{K}+$ & $\mathrm{Mg} 2+$ & $\mathrm{Na}$ & Cond \\
\hline $\mathrm{NO} 2$ & 1 & & & & & & & & & & & & \\
\hline NH4 & 0,236 & 1 & & & & & & & & & & & \\
\hline $\mathrm{PO} 4$ & 0,104 & 0,246 & 1 & & & & & & & & & & \\
\hline NO3 & $-0,624$ & $-0,273$ & $-0,074$ & 1 & & & & & & & & & \\
\hline $\mathrm{pH}$ & $-0,277$ & $-0,047$ & 0,118 & $-0,133$ & 1 & & & & & & & & \\
\hline $\mathrm{T}^{\circ} \mathrm{C}$ & $-0,230$ & 0,077 & 0,099 & 0,280 & 0,384 & 1 & & & & & & & \\
\hline SO4 & $-0,383$ & $-0,054$ & 0,268 & 0,494 & 0,283 & 0,586 & 1 & & & & & & \\
\hline $\mathrm{Cl}-$ & $-0,218$ & 0,428 & 0,224 & 0,259 & $-0,165$ & 0,259 & 0,522 & 1 & & & & & \\
\hline $\mathrm{Ca} 2+$ & $-0,334$ & $-0,102$ & $-0,065$ & 0,378 & 0,725 & 0,504 & 0,589 & 0,059 & 1 & & & & \\
\hline $\mathrm{K}+$ & $-0,484$ & $-0,142$ & $-0,072$ & 0,590 & $-0,029$ & 0,461 & 0,705 & 0,563 & 0,370 & 1 & & & \\
\hline $\mathrm{Mg} 2+$ & $-0,093$ & $-0,171$ & 0,115 & 0,248 & 0,559 & 0,529 & 0,564 & $-0,007$ & 0,811 & 0,316 & 1 & & \\
\hline $\mathrm{Na}$ & $-0,450$ & $-0,156$ & $-0,247$ & 0,636 & $-0,345$ & 0,229 & 0,533 & 0,614 & 0,046 & 0,814 & $-0,029$ & 1 & \\
\hline Cond & $-0,562$ & $-0,208$ & $-0,247$ & 0,757 & $-0,129$ & 0,346 & 0,662 & 0,559 & 0,351 & 0,889 & 0,218 & 0,940 & 1 \\
\hline
\end{tabular}


F. KANOHIN Epse OTCHOUMOU et al. / Int. J. Biol. Chem. Sci. 11(5): 2495-2509, 2017
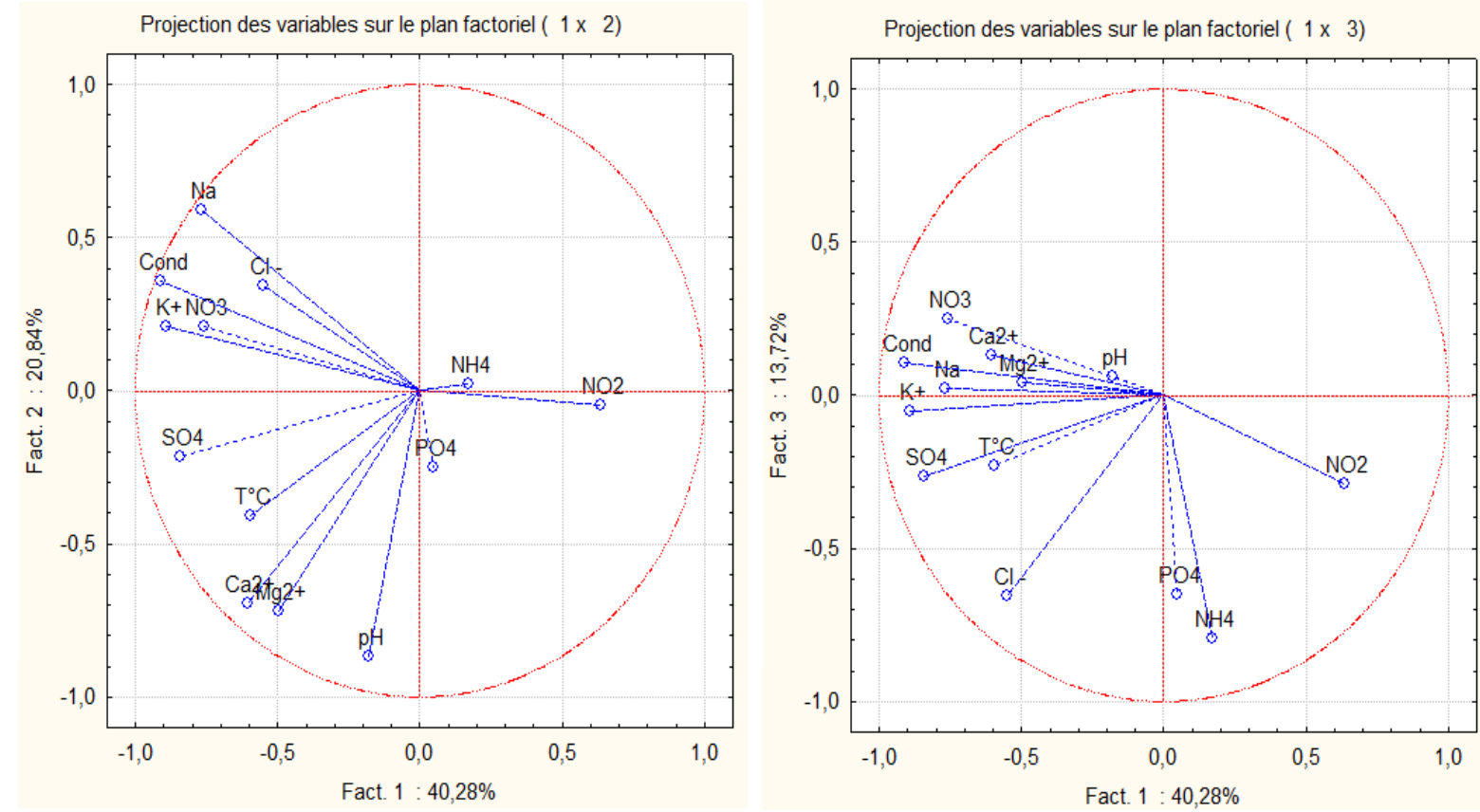

Figure 4 : Cercle des corrélations F1-F2 et F1-F3.

Projection des ind. sur le plan factoriel ( $\left.\begin{array}{ll}1 \times 2 & 2\end{array}\right)$

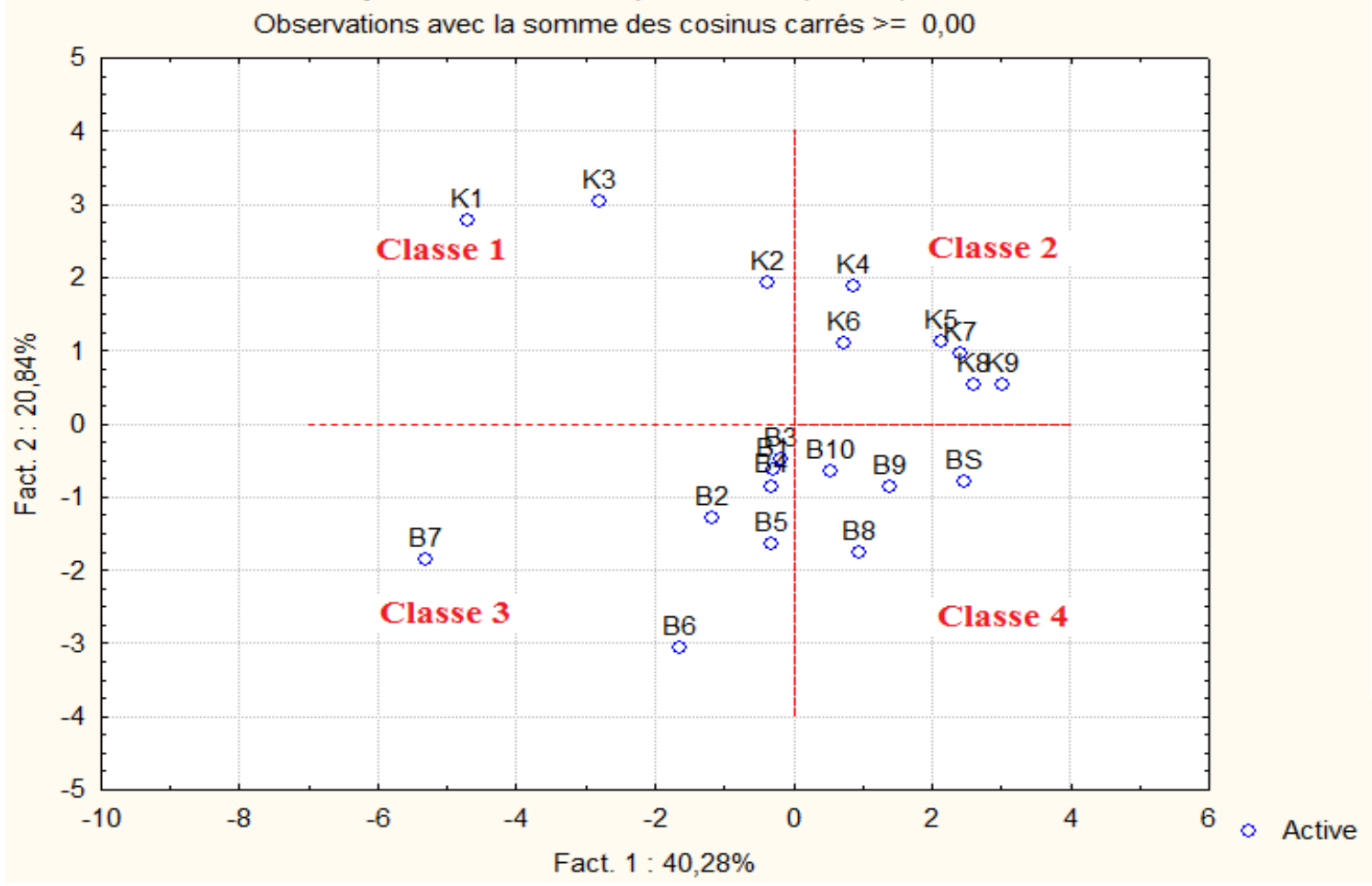

Figure 5 : Représentation graphique de la carte factorielle des puits de Brègbo et de Koffikro. 


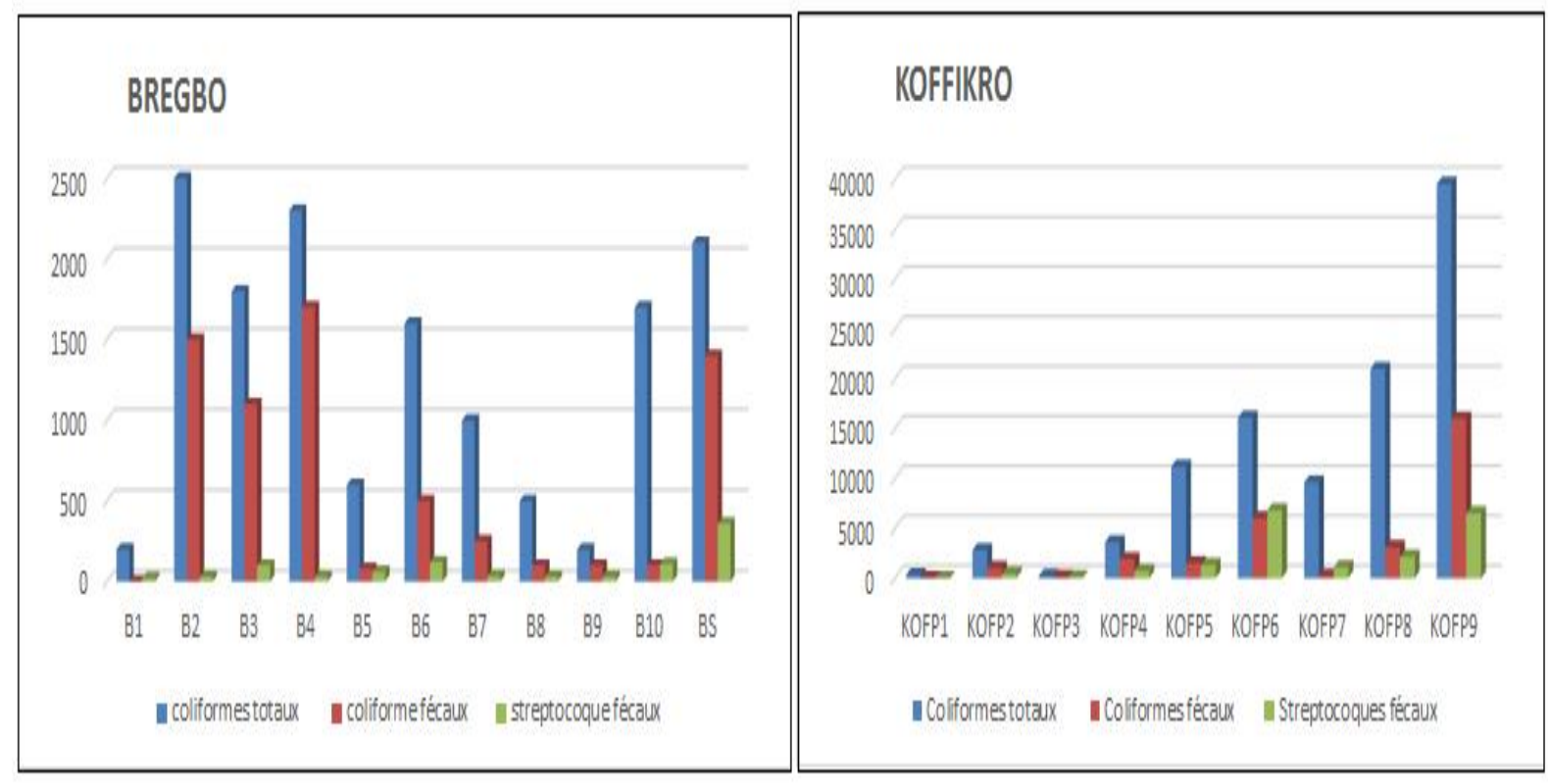

Figure 6 : Histogramme de trois germes recherchés dans les puits de Brègbo et Koffikro.

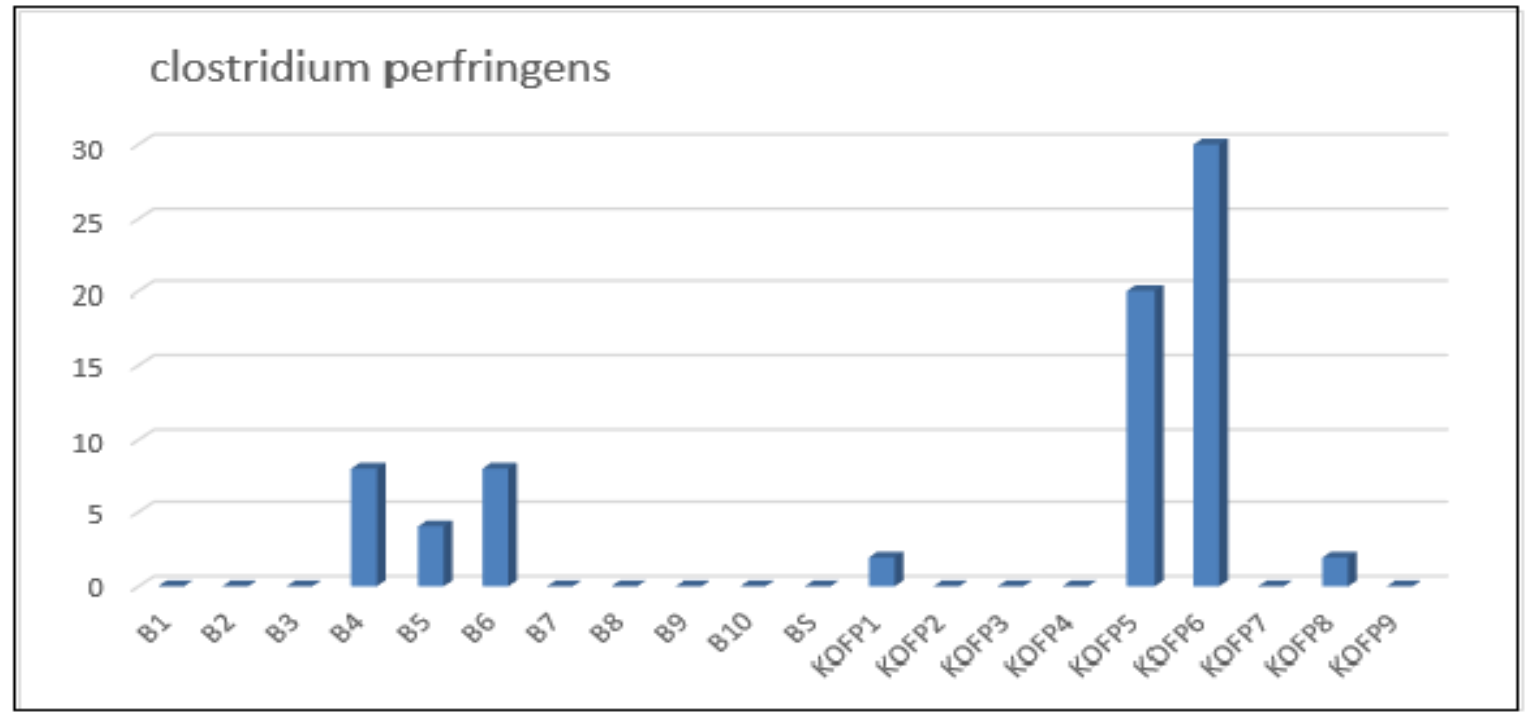

Figure 7 : Variation des taux de clostridium perfringens dans les différents puits. 
Tableau 4 : Charge moyenne des bactéries dans les de puits de Brègbo et de Koffikro.

\begin{tabular}{lcc}
\hline & \multicolumn{2}{c}{ Zones d'études } \\
\cline { 2 - 3 } Charges en UFC/100 ml & Brègbo & Koffikro \\
\hline Coliformes totaux & 1318 & 11600 \\
Coliformes fécaux & 620 & 3311 \\
Streptocopes fécaux & 84 & 2113 \\
Clostridium perfringens & 10 & 30 \\
\hline
\end{tabular}

\section{DISCUSSION}

La qualité d'une eau souterraine est caractérisée par les concentrations en ions $\mathrm{NH}_{4}^{+}, \mathrm{PO}_{4}^{3-}, \mathrm{SO}_{4}{ }^{2-}$ et $\mathrm{NO}_{3}^{-}$. Les normes de l'OMS (Organisation Mondiale de la Santé) et de l'union européenne (UE) définissent les valeurs limites de $\mathrm{NH}_{4}^{+}, \mathrm{PO}_{4}{ }^{3-}, \mathrm{SO}_{4}{ }^{2-}$ et $\mathrm{NO}_{3}{ }^{-}$ qui sont respectivement de $0,5 \mathrm{mg} / \mathrm{l}, 0,005$ $\mathrm{mg} / \mathrm{l}, 50 \mathrm{mg} / \mathrm{l}$ et $250 \mathrm{mg} / \mathrm{l}$ dans une eau de boisson. Les valeurs élevées des concentrations en ammonium sont observées dans le village de Koffikro avec des pics de $3,9 \mathrm{mg} / \mathrm{l}$. Ce qui est nettement supérieur à la valeur seuil recommandée par l'OMS $(0,5$ $\mathrm{mg} / \mathrm{l}$ ) pour une eau de boisson. La présence de l'ammonium dans l'eau est le signe d'un processus de dégradation incomplet de la matière organique. Il provient également de l'excrétion des organismes vivants et de la réduction de l'azote organique au cours de la biodégradation des déchets, sans négliger les apports direct d'origine domestique et agricole (Derwich et al., 2010). C'est donc un excellent indicateur de la pollution de l'eau. Les pics d'ammonium observés à Koffikro pourraient se justifier par la présence des porcheries. Selon Dupond (2009), l'élevage est l'une des plus grandes sources de polluant des eaux souterraines à travers les déjections des animaux (urines et excréments). L'élevage à Koffikro contribue de façon directe par les rejets de lisiers, à la contamination des eaux en ammonium. La mauvaise gestion des déjections animales et les élevages intensifs provoquent le lessivage des nitrates et des agents pathogènes dans la nappe aquifère, qui met souvent en péril les réserves d'eau potable (FAO, 2005). On note la présence de phosphate dans tous les puits échantillonnés.
Les concentrations en phosphate varient entre $0,004 \mathrm{mg} / \mathrm{l}$ et $0,29 \mathrm{mg} / \mathrm{l}$. Plus de $80 \%$ des puits ont des concentrations en phosphate supérieur à la valeur seuil de l'OMS $(0,005 \mathrm{mg} / \mathrm{l})$. Ces teneurs élevées semblent être liées à une potentielle intrusion de la nappe phréatique par les eaux de la lagune, les eaux de ruissellement agricole riches en engrais (FAO, 2005) et aussi de la proximité des fosses septiques. Les teneurs en nitrate des puits à Brègbo et Koffikro sont inférieurs à $50 \mathrm{mg} / \mathrm{l}$. Ces deux localités n'abritent pas d'exploitations agro-industrielles utilisant d'importantes quantités d'engrais. Car des valeurs supérieures indiquent une utilisation excessive de fertilisants utilisés en agriculture (Chapman et Kimstach, 1996).

De fortes valeurs de conductivité ont été constatées dans les puits $\mathrm{B} 7, \mathrm{~K} 1, \mathrm{~K} 2$ et $\mathrm{K} 3$ dont les valeurs respectives sont de 764 $\mu \mathrm{S} / \mathrm{cm}, 860 \mu \mathrm{S} / \mathrm{cm}, 495 \mu \mathrm{S} / \mathrm{cm}$ et $744 \mu \mathrm{S} / \mathrm{cm}$. La valeur de la conductivité est influencée par divers facteurs naturels et anthropiques dont la géologie du bassin versant (la composition des roches), les apports d'eau souterraine et les apports d'eau contaminée provenant des activités humaines (Hade, 2002) car les rejets contaminés augmentent aussi la conductivité de l'eau (WASC, 2003). Les résultats de l'ACP révèlent une corrélation entre la conductivité et les ions en solution. Ces corrélations reflètent l'influence de chaque paramètre dans la minéralisation de l'eau souterraine de Bingerville. Cette corrélation de la conductivité avec ces ions majeurs rend compte de la minéralisation ou du phénomène de l'hydrolyse des minéraux (Ahoussi et al., 2010). Il faut noter que la conductivité décrit les sels inorganiques présents en solution dans 
l'eau. La corrélation entre le magnésium et le calcium $(\mathrm{r}=0,811)$ reflète la dissolution des roches liée au temps de séjour de l'eau dans l'aquifère (Saadek, 2011; Ahoussi et al., 2010). Les faibles concentrations de calcium et de magnésium indiquent que nous sommes en présence d'eaux douces acides et subissant des intrusions d'eaux usées. La corrélation entre le potassium et le sodium $(r=0,814)$ et entre les ions chlorures et le sulfate $(r=0,522)$ mettent en évidence la salinisation due à la proximité des latrines et de l'intrusion des eaux saumâtres dans la nappe phréatique (Dibi et al., 2014).

Les résultats des différentes analyses montrent que ces eaux sont impropres à la consommation humaine, car les normes de l'OMS exigent l'absence totale de germes de contamination fécale dans les eaux destinées à la boisson (WHO, 2008). La ville de Bingerville exploite pour son alimentation en eau potable la nappe profonde à travers la SODECI (Société de distribution d'eau de Côte d'Ivoire). Cependant en milieu rural, les localités exploitent la nappe phréatique à travers les puits et les sources. L'accès aux services d'assainissement et de distribution d'eau potable étant inexistant, l'assainissement des excréta est réalisé en grande majorité par des latrines traditionnelles à fonds perdu dont la fosse réalisée par des puisatiers de fortune atteint généralement la nappe phréatique et constitue un risque de contamination microbiologique des eaux souterraines (Jourda et al., 2006 ; Yapo et al., 2010 ; Mbalawa et al., 2010). Ce que confirment d'ailleurs, les résultats des analyses microbiologiques qui traduisent une forte contamination des eaux de puits par des germes d'origine fécale. Les eaux de la localité de Koffikro ont une charge bactérienne très élevée en comparaison aux eaux de Brégbo. Le village de koffikro renferme de nombreuses porcheries et selon Dupond (2009), l'élevage est l'une des plus grandes sources de polluant des eaux souterraines à travers les déjections des animaux (urines et excréments). La charge microbienne importante dans les eaux de puits analysées pourrait se justifier par un certain nombre de facteurs inhérents aux activités agro-pastorales autour des puits, un défaut d'aménagement de ces puits, la proximité des sources de pollution telles que les ordures ménagères, les latrines et le non-respect des règles d'hygiène élémentaire par les utilisateurs (Mbawala et al., 2010).

Les puits étudiés ont des profondeurs inferieures à $10 \mathrm{~m}$ avec des niveaux piézométriques qui oscillent entre $1,36 \mathrm{~m}$ et $8,02 \mathrm{~m}$. Cette proximité de l'eau souterraine par rapport à la surface topographique renforce le caractère vulnérable de la plupart des puits des différentes localités de Bingerville. Les études menées par M'bawala et al. (2010) sur les eaux de puits à Dang au Cameroun, confirment cette assertion selon laquelle la profondeur des puits et la distance entre ceux-ci et les latrines influencent la prolifération des germes dans l'eau.

Les analyses microbiologiques montrent la présence des bactéries de types coliformes totaux, coliformes fécaux, streptocoques fécaux et Clostridium Perfringens dans les eaux de puits de Brègbo et Koffikro. Tous les puits visités sont contaminés par les germes fécaux sauf le puits B1. Ce qui traduit le défaut d'assainissement $\mathrm{du}$ milieu qui expose les ressources en eaux à de fortes contaminations de bactéries d'origine fécale (Youmbi et al., 2012). Selon Guessoum et al. (2014), la présence des spores des anaérobies sulfito-réducteurs dans une eau naturelle fait penser à une contamination fécale et en l'absence de bactéries Coliformes, à une contamination ancienne. Elles sont très persistantes et leur présence est un bon indicateur de la vulnérabilité des aquifères et des puits (Ayad et al., 2016). La présence des Clostridium sulfito-réducteurs est très significative d'une pollution fécale ancienne ou intermittente. La présence de ces bactéries pourrait être due à la mauvaise protection des puits (puits à ciel ouvert), la pollution avoisinante (élevage des bétails, existence des fosses septiques et des latrines) et l'absence d'un réseau d'assainissement (Yapo et al., 2010). Les eaux de ces puits sont non potables car les normes de l'OMS exigent 
l'absence de ces bactéries dans les eaux destinées à la consommation (WHO, 2008).

\section{Conclusion}

Les données recueillies au cours de cette étude ont permis de dresser un portrait de la qualité physico-chimique et microbiologique des eaux de puits à usages domestiques des localités de Brègbo et Koffikro. Les eaux des puits échantillonnées sont de mauvaise qualité et ne satisfont pas aux normes de qualité de l'eau potable. Les paramètres les plus menaçants restent le phosphate $\left(\mathrm{PO}_{4}{ }^{2-}\right)$ et l'ammonium $\left(\mathrm{NH}_{4}{ }^{+}\right)$dont les moyennes demeurent fortement élevées. Sur le plan bactériologique, $80 \%$ des eaux de puits analysées sont souillées par les germes de contamination fécale et ne doivent pas être consommées sans traitement préalable. Cette pollution trouve très probablement ses origines dans l'absence des services d'assainissements et de collecte des ordures ménagères.

\section{CONFLITS D'INTERETS}

Les auteurs déclarent qu'ils n'ont pas d'intérêts concurrents.

\section{CONTRIBUTIONS DES AUTEURS}

Les campagnes d'échantillonnages ont vu la participation de OBY, ACB et FKEO. La rédaction du manuscrit a vu la contribution de OBY, BD et FKEO.

\section{REFERENCES}

AFNOR (Association Française de Normalisation). 1997. Qualité de l'Eau: Terminologie, Echantillonnage et Evaluation des Méthodes (Tome 1, $3^{\mathrm{e}}$ édn). AFNOR : Paris, France.

Ahoussi KE, Soro N, Koffi YB, Soro G, Biemi J. 2010. Origine de la minéralisation des eaux des aquifères discontinus sous couvert forestier de la zone Sud de la Côte d'Ivoire : cas de la région d'Abidjan-Agboville. Int. J. Biol. Chem. Sci., 4(3): 782-797, DOI: http://ajol.info/index.php/ijbcs

Ambarref M, Saadia Aassine, Bernoussi AS, Haddouchi BY. 2007. Cartographie de la vulnérabilité à la pollution des eaux souterraines: application à la plaine du Gharb (Maroc). Revue des Sciences de l'Eau, 20(2): 185-199, DOI : 10.7202/015812ar

ACI (Atlas Côte d'Ivoire). 1986. Atlas de Côte-d'Ivoire. Ministère du plan de Côted'Ivoire. Office de la recherche scientifique et technique outre-mer. Institut de géographie tropicale. Université d'Abidjan, 67 p.

Ayad W, Kahoul M. 2016. Evaluation de la qualité physico chimique et bactériologique des eaux de puits dans la région d'El Harrouch (N.E Algérie). $J$. Mater. Environ. Sci., 7(4) : 1288-1298, DOI : http://www.jmaterenvironsci.com

Bouroche JM, Saporta G. 1992. L'Analyse des Données (Collection Que sais-je ? $\mathrm{n}^{\circ}$ $854,5^{\text {ème }}$ édn). Presses Universitaires de France : Paris ; 1-27

Caillez JP, Pages. 1976. Introduction à l'Analyses des Données. SMASH: Paris, 616.

Coulibaly L, Diomandé D, Coulibaly A, Gourène G. 2004. Utilisation des ressources en eaux, assainissement et risques sanitaires dans les quartiers précaires de la commune de Port-Bouët (Abidjan; cote d'ivoire). Vertigo - La Revue en Sciences de l'Environnement, 5(3): 11, DOI : 10.4000/vertigo.3299

Chapman D, Kimstach V. 1996. Selection of Water Quality Variables. Water Quality Assessments: a Guide to the Use of Biota, Sediments and Water in Environment Monitoring. Chapman edition: London; 59-126.

Derwich E, Benaabidate L, Zian A, Sadki O, Belghity D. 2010. Caractérisation physico-chimique des eaux de la nappe alluviale du haut sebou en aval de sa confluence avec oued fes. Larhyss Journal, 08: 101-112, DOI: https://www.academia.edu/26186009/

Dibi B, Konan KS, Konan-Weidhet AB, Savané I, Gnakri D. 2014. Analysis of the impact of anthropic activities on the water chemistry of weathered layer aquifer of M'bahiakro locality (Center of 
Côte d'Ivoire). American Journal of Engineering Research (AJER), 3(1): 4349, DOI: www.ajer.org

Dupont G. 2009. Le développement fulgurant de l'aquaculture devrait continuer. Le Monde.

El Asslouj J, Kholtei S, El Amrani-Paaza N, Hilali A. 2007. Impact des activités anthropiques sur la qualité des eaux souterraines de la communauté Mzamza (Chaouia, Maroc). Rév. Sci. Eau, 20(3): 306-321, DOI: 10.7202/016505ar

FAO (Food and Agriculture Organization). 2005. Pollution from Industrialized Livestock Production. FAO, Livestock Policy Briefs: Rome.

Fofana F. 2005. Evaluation et cartographie de la vulnérabilité à la pollution de la nappe d'Abidjan selon les méthodes Drastic et God. Mém. DEA, Univ. Abobo-Adjamé, Côte d'Ivoire, $72 \mathrm{p}$.

Guessoum H, Benbrahim F, Halilat MT, Laouar F, Bensalama M, Darem S. 2014. Pollution Biologique des Eaux Phréatiques de la Région de Ghardaia (Cas de Sebseb). Journal of Advanced Research and Science and Technology, 3: 35-43, DOI: http://www.jarst.info/

Hade A. 2002. Nos Lacs - Les Connaître pour Mieux les Protéger. Éditions Fides ; 360 p.

INS (Institut national de la statistique). 2014. Recensement général de la population et de l'habitat de Côte d'Ivoire. Rapport de synthèse, volume des fiches monographiques des localités : région des lagunes. INS Abidjan, $50 \mathrm{p}$.

Jang CS, Chen JS, Lin YB, Liu CW. 2012. Characterizing hydrochemical properties of springs in Taiwan based on their geological origins. Environ Monit Assess, 184: 63-75, DOI 10.1007/s10661-0111947-4.

Jourda JP, Kouamé KJ, Saley MB, Kouadio $\mathrm{BH}$, Oga YS. 2006. Contamination of the Abidjan Aquifer by Sewage: An Assessment of Extent and Strategies for
Protection In Groundwater Pollution in Africa, Yongxin X, Brent U (eds). Taylor.

Mbawala A, Abdou, Ngassoum MB. 2010. Evaluation de la pollution physicochimique et microbienne des eaux de puits de Dang-Ngaoundéré (Cameroun). Int. J. Biol. Chem. Sci., 4(6): 1962-1975. DOI: http://ajol.info/index.php/ijbcs

Rodier JC, Bazin JF, Busby RW, Lee BB. 1996. L'Analyse de l'Eau (8 $8^{\mathrm{èm}}$ édn). DUNOD: Paris, France.

Sadek A. 2011. Hydrochimie et faciès géochimiques des eaux souterraines, Plaine de Bekaa. Hydrological Sciences Journal, 56(2) : 334-348. DOI: 10.1080/02626667.2011.559331.

Tagini B. 1971. Esquisse structurale de la Côte-d'Ivoire. Essai de géotectonique régionale. Thèse de Doctorat d'Université de Lausanne (Suisse). Bulletin, SODEMI, ${ }^{\circ}$ 5, $302 \mathrm{p}$.

WHO (World Health Organization). 2008. Guidelines for Drinkink-Water Quality, $\left(3^{\text {rd }}\right.$ edn, vol 1). Recommandations: Geneva.

Yapo OB, Mambo V, Seka A, Ohou MJA, Konan F, Gouzile V, Tidou A. S, kouame KV et Houenou P. 2010. Evaluation de la qualité des eaux de puits à usage domestique dans les quartiers défavorisés de quatre communes d'Abidjan (Côte d'Ivoire) : Koumassi, Marcory, Port-Bouet et Treichville. Int. J. Biol. Chem. Sci., 4(2): 289-307. DOI: http://ajol.info/index.php/ijbcs

Youmbi JGT, Feumba R, Njitat VT, Marsily G, Ekodeck GE. 2013. Pollution de l'eau souterraine et risques sanitaires à Yaounde au Cameroun. Comptes Rendus Biologies, 336(5-6): 310-316. DOI: 10.1016/j.crvi.2013.04.013

WASC (Waterwatch Autralia Steering Committee). 2003. Environment Australia. Waterwatch Australia National Technical Manual; 156 p. 